Musées, Patrimoine et Culture scientifiques et techniques

$121 \mid 2009$

janvier - février 2009

\title{
Un inventaire du patrimoine géologique pour la
} France

\section{Patrick De Wever}

\section{(2) OpenEdition \\ Journals}

Édition électronique

URL : http://journals.openedition.org/ocim/267

DOI : 10.4000/ocim.267

ISSN : 2108-646X

Éditeur

OCIM

Édition imprimée

Date de publication : 1 janvier 2009

Pagination : 12-18

ISSN : 0994-1908

Référence électronique

Patrick De Wever, "Un inventaire du patrimoine géologique pour la France », La Lettre de I'OCIM [En ligne], 121 | 2009, mis en ligne le 01 janvier 2011, consulté le 30 avril 2019. URL : http:// journals.openedition.org/ocim/267 ; DOI : 10.4000/ocim.267 
mais le plus souvent à Antoine de Saint-Exupéry. Pourtant l'association qui perpétue la mémoire de cet écrivain en dénie cet octroi. Admettons alors que cette formule appartient désormais au patrimoine commun, elle est devenue un adage.

La notion de patrimoine est bien intégrée à notre culture. Les notions de conservation et de transmission y sont associées ainsi que le souci, en général, de ne pas dilapider l'héritage. Historiquement, l'approche patrimoniale est liée à la réalisation des hommes, avec la protection des œuvres artistiques. On citera le tour de France, au début du XIX ${ }^{\mathrm{e}}$ siècle, de Prosper Mérimée, alors inspecteur général des Monuments Historiques, qui a effectué le premier un classement des monuments historiques. Dans la nature, la première zone protégée l'a été pour des raisons... artistiques. En effet, en forêt de Fontainebleau, sous l'action de peintres de Barbizon (Courbet, Millet, Rousseau, La Peña...) est ajournée la coupe de vieilles futaies pour le remplacement des chênes par des résineux dès 1836. La première réserve y est créée en 1861.

\section{Tout site, tout objet dépend-il du patrimoine?}

Philippe Billet (2002) a tenté une définition de la notion de patrimoine géologique dans son guide juridique du patrimoine géologique : «tous les témoins de l'histoire de la Terre qui participent de la connaissance des événements physiques et biologiques qui ont marqué notre planète ». Est-ce à dire que tous les affleurements, sources de nos informations, sont patrimoine? Jules Gosselet a écrit «dans notre vie de géologue, chaque fois que nous rencontrons un rocher, nous lui demandons son nom, son âge, pourquoi il est là, comment il s'est formé, et pour ne pas oublier ses réponses, nous en rapportons un fragment dans nos collections ». C'est ainsi qu'il a constitué le patrimoine du musée de Géologie de Lille qui porte son nom et auquel il se voua jusqu'à en mourir (1). Tous les échantillons des tiroirs sont donc patrimoine. Pourtant chacun sait que dans tout héritage, il y a à trier et chacun trie, selon son évaluation, ce qu'il va conserver et transmettre et ce qu'il ne retiendra pas. De la même façon que le naturaliste du vivant a su distinguer la nature ordinaire, qui mérite attention, de la nature exceptionnelle, qui focalise les actions conservatoires, le géologue doit distinguer dans son environnement minéral les objets géologiques ordinaires de ceux, remarquables, dont il se sent responsable pour la transmission aux générations futures.
Un objet géologique remarquable est distingué pour des motifs très différents : son intérêt scientifique, pédagogique, historique, ou pour sa rareté, son exemplarité, sa représentativité, ou encore pour son état exceptionnel de conservation, ou encore de sa qualité esthétique, voire parce qu'il est associé à un autre patrimoine. Parfois, il ne l'est qu'aux yeux des seuls spécialistes : un stratotype par exemple n'apparaît le plus souvent que comme un banal talus, dans d'autres cas sa lisibilité est évidente pour tous (voir encadré ci-contre).

Le patrimoine se trouve aussi bien sur le terrain (patrimoine in situ), que dans les collections (patrimoine ex situ) privées ou publiques (universités, musées) mais aussi dans certains documents (écrits, photographies, cartes, dessins...). Tout cela est mémoire, mais tout ne peut être conservé. Des choix sont à faire entre les trésors qui ont le plus de valeur. Patrimoine, valeur, trésor... chacun de ces vocables contient des acceptions bien différentes.

\section{Une mémoire d'avant la mémoire de l'Homme}

"Comme un vieil arbre garde la mémoire de sa croissance et de sa vie dans son tronc, la Terre conserve la mémoire du passé... une mémoire inscrite dans les profondeurs et sur la surface, dans les roches, les fossiles et les paysages, une mémoire qui peut être lue et traduite.

Aujourd'hui, les hommes savent protéger leur mémoire: leur patrimoine culturel. À peine commence-t-on à protéger l'environnement immédiat : notre patrimoine naturel. Le passé de la Terre n'est pas moins important que le passé de l'Homme. Il est temps que l'Homme apprenne à protéger et, en protégeant, apprenne à connaître le passé de la Terre, cette mémoire d'avant la mémoire de l'Homme qui est un nouveau patrimoine : le patrimoine géologique. " (articles 6 et 7 de la Déclaration internationale des Droits de la Mémoire de la Terre, Digne-les-Bains, 1991).

La conservation du patrimoine passe nécessairement par un statut juridique pour les objets géologiques remarquables. Philippe Billet a magistralement éclairé la situation : " toute chose a un statut au regard $d u$ droit de propriété. Les éléments du patrimoine géologique n'échappent pas à cette règle ». «Un minéral 


\section{Des stratotypes pour une échelle des temps géologiques}

L'échelle des temps géologiques est immense. Il est donc nécessaire, pour s'y retrouver de disposer de quelques repères tout comme on a besoin de dates $(732,1515 \ldots)$ pour se situer dans l'échelle des temps historiques. Une échelle peut être subdivisée de deux façons : par les intervalles entre ses barreaux, et par ses barreaux.

Dans un premier temps, les géologues industriels, issus de l'École des Mines pour la plupart tel Élie de Beaumont, ont utilisé le type de roche pour distinguer les différents niveaux, procédé qui répondait à une logique d'exploitation. Ils avaient remarqué que telle période était favorable à tel type de roche. Ils ont alors baptisé cette période à partir du nom de cette roche. Ainsi sont apparus le Carbonifère, qui portait du charbon, le Crétacé, l'époque de la craie... D'autres géologues, relevant plutôt du monde académique, tel Alcide d'Orbigny, s'intéressaient plus spécifiquement aux fossiles. Ils avaient observé qu'un groupe de strates contenait un certain ensemble de fossiles, différent de celui des strates supérieures et inférieures. Ils avaient alors utilisé ce critère paléontologique pour définir le groupe de strates qui représentait une certaine durée. La période de temps correspondant était généralement baptisée du nom du lieu où avait été définies ces couches : ainsi trouve-ton l'Aptien près d'Apt, le Lutétien en région parisienne ou encore le Séquanien en Bourgogne... L'endroit où ont été décrites les strates de référence est appelé : stratotype, pour les strates types. Les stratotypes sont des références, locales, nationales voire internationales et méritent donc, au même titre que le mètre étalon, d'être conservées. À la différence du mètre

cependant, elles doivent être conservées dans la nature, précisément là où elles ont été définies. Ces sites qui méritent protection relèvent du patrimoine scientifique mondial.

L'Europe occidentale était au XIX siècle un endroit privilégié pour les scientifiques, aussi beaucoup de ses localités portent des noms d'étages géologiques. La France possède ainsi une quarantaine de stratotypes qui exposent cette valeur de référence mondiale, pourtant seuls quelques-uns d'entre eux sont protégés

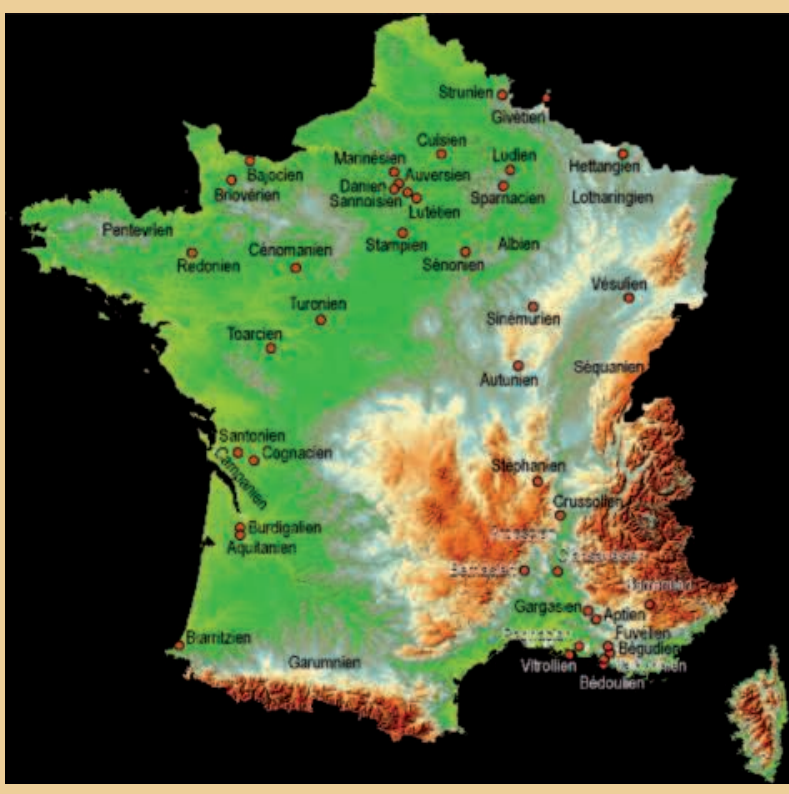

Localisation des stratotypes en France (c) Patrick De Wever
(12 au total), et rares sont

les localités - comme Barrème dans les Alpes de Haute-Provence - qui ont conscience d'être le toponyme d'un étage géologique.

Parce qu'ils ont été définis en fonction des fossiles, les stratotypes sont généralement des localités particulièrement riches en restes paléontologiques et correspondent donc souvent à des dépôts proches du littoral puisque c'est là que les restes d'organismes sont les plus fréquents. Le problème est que ces endroits sont aussi très sensibles à l'érosion ou au moins à une absence de sédimentation ce qui se traduit en terme d'enregistrement sédimentaire par un hiatus : les stratotypes sont riches en fossiles et en lacunes! Pour remédier à cette situation paradoxale les stratigraphes ont alors choisi de ne plus définir les étages par les espaces entre les barreaux mais par les barreaux eux-mêmes c'est-à-dire qu'un événement marque une limite. En général il s’agit de l'apparition d'un fossile. On est ainsi passé de la notion de " stratotype de couche » à celle de «stratotype de limite » et cette limite est marquée par un clou d'or (plus prosaïquement représenté par un clou en acier inoxydable). S'il y avait une quarantaine d'étages définis en France, il n'y a que cinq stratotypes de limite définis en France (Campanien-Maastrichtien à Tercis, Landes ; Albien-Cénomannien au Mont-Risou, Hautes-Alpes ; Famennien-Tournaisien à La Serre, Cabrières Montagne Noire ; FrasnienFamennien à Coumiac, Montagne Noire ; Givetien-Frasnien au col du Puech de la Suque, Saint-Nazairede-Ladarez, Montagne Noire). Actuellement, l'Europe et la France, en particulier, semblent se désintéresser de cette discipline à la différence de la Chine. La conséquence directe est que les noms chinois se multiplient dans l'échelle internationale. 


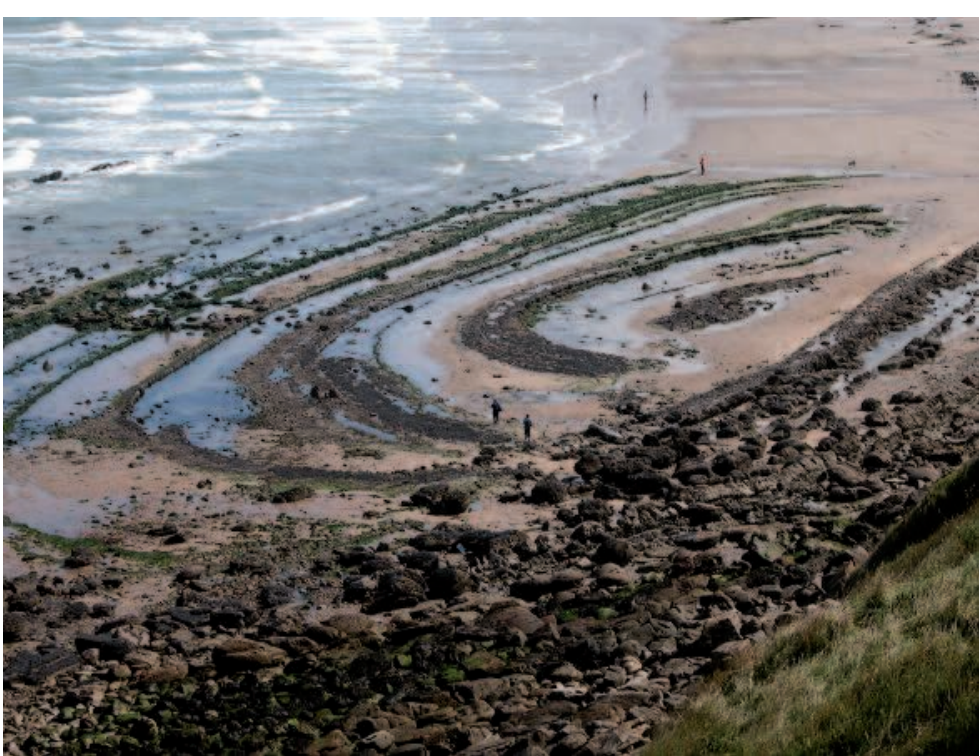

Au Cap Gris Nez, dans le Boulonnais, une structure anticlinale en voûte a été érodée par la mer et laisse alors apparaître ses différents niveaux emboîtés, le plus ancien étant au centre. Cette structure apparaît à une échelle qui n'est généralement visible que sur les cartes géologiques. (c) Patrick De Wever

[ou une roche] ou un fossile contenu dans le sol ou qui repose en surface tout en restant rattaché au sol est un bien immeuble. Il devient bien meuble après son détachement ou son extraction. » Ainsi au regard du Code civil ils ont un propriétaire : "le patrimoine géologique appartient, par principe, au propriétaire $d u$ sol qui le renferme ou qui le supporte. Il bénéficie ainsi du régime de la propriété privée. » Cette possession est claire, elle est étayée par une bonne jurisprudence (Billet, 2002).

La protection de sites géologiques (paléontologiques ou minéralogiques) présentant un intérêt particulier passe par l'identification de sites et leur inscription sur des listes comme cela existe pour la faune, la flore, les zones humides, les Zones Naturelles d'Intérêt Écologique Faunique ou Floristique (ZNIEFF). Quel que soit le droit applicable, la protection du patrimoine passe par une prise de conscience de sa fragilité, éveillée par des actions de sensibilisation, pour une responsabilisation qui ne soit pas vécue comme une contrainte.

Quand on parle de patrimoine géologique, ce dernier vocable doit être pris dans son acception large geo-logos, tout ce qui parle de la Terre, et non dans une acception étriquée où l'on distinguerait la paléontologie, de la minéralogie, de la sédimentologie, de la stratigraphie...

\section{Quand la Terre rejoint la nature dans la loi}

Il a fallu attendre la loi relative à la protection de la nature de 1976 (la création d'un ministère chargé de l'Environnement ne date que de 1971) pour qu’apparaisse la notion de patrimoine naturel. Bien que cette loi - qui crée officiellement le concept de réserve naturelle - envisage la protection possible « des gisements de minéraux et de fossiles » et des "formations géologiques, géomorphologiques ou spéléologiques remarquables », force est de constater que durant les trente dernières années du XXe siècle, les naturalistes biologistes ont été beaucoup plus actifs que les géologues plus excités par la nouvelle tectonique globale.

La prise de conscience officielle date de 1991, lors du premier symposium international (une trentaine de nationalités) sur la protection du patrimoine géologique à Digne où a été proclamée la Déclaration internationale des Droits de la Mémoire de la Terre, publiée par la Société géologique de France en 1994. Quelques temps forts marquent une période de maturation : les premières journées nationales du patrimoine géologique en 1997, suivies des deuxièmes (1999), troisièmes (2002) et quatrièmes (2008); la création, par le ministère chargé de l'Environnement, de la Conférence permanente du Patrimoine géologique (CPPG) en 1998 qui rassemble des représentants du Muséum national d'Histoire naturelle, des Réserves naturelles de France, des musées, des amateurs, du Bureau de Recherches géologiques et minières (BRGM) ainsi que des intuitu personae. Ce n'est que depuis 2002 qu'est officiellement acquise la reconnaissance du patrimoine géologique au même niveau que le patrimoine biologique au sein du patrimoine naturel. Jusque-là, la Terre n'appartenait pas à la nature du point de vue juridique ! En effet, la nouvelle rédaction de l'article L411-5 du Code de l'Environnement, issue de la loi du 27 février 2002 relative à la démocratie de proximité, donne corps à l'inventaire du patrimoine naturel sur l'ensemble du territoire national. Pour la première fois il comprend de manière explicite les richesses "géologiques, minéralogiques et paléontologiques».

Dans cette loi on lit que "l'inventaire du patrimoine naturel est institué pour l'ensemble $d u$ territoire national... L'État en assure la conception, l'animation et l'évaluation... Ces inventaires [patrimoine naturel] sont conduits sous la responsabilité scientifique $d u$ Muséum national d'Histoire naturelle... Il est institué dans chaque région un Conseil scientifique régional du Patrimoine naturel (CSRPN) (2)... Il couvre toutes 
les disciplines des Sciences de la Vie et de la Terre». L'État, maître d'ouvrage, était représenté par la Direction de la Nature et des Paysages (DNP). Cette DNP fait aujourd'hui partie de la Direction de l'Eau et de la Biodiversité (DEB). Il a confié à la Conférence permanente du Patrimoine géologique le soin de finaliser la politique nationale de réalisation de l'inventaire, dans ses composantes administratives et techniques. Quelques contraintes fortes s'imposaient :

- la compatibilité des données avec les inventaires faune, flore et ZNIEFF,

- une homogénéité au niveau national,

- une technique de saisie relativement légère afin que le mouvement associatif, dont les moyens financiers sont souvent limités, puisse participer.

Pour faire face à ces prérequis, la CPPG s'est donc basée sur l'expérience des Réserves naturelles de France et sur celle des ZNIEFF.

Afin de permettre une réalisation homogène et cohérente sur l'ensemble du territoire, l'inventaire doit être mené sur la base d'une méthode élaborée au niveau national par les différents partenaires intéressés, à partir de l'expérience des inventaires faune, flore, ZNIEFF... et de celle des Réserves naturelles. Nous avons repris une démarche de même type que celle des ZNIEFF qui a fait ses preuves en termes de sauvegarde et dans les démarches d'aménagement (certains sites de supermarchés ne se sont pas installés pour préserver des sites). La méthode a été testée avec les données de la Région Bretagne qui, à l'instar d'autres départements ou régions, avait commencé un inventaire. Pendant la phase pilote, les rubriques et le mode de saisie ont été affinés. Afin de faciliter l'harmonisation des données, un logiciel de saisie Géotope, a été mis en œuvre par le Bureau de Recherches géologiques et minières, selon les instructions de la CPPG.

La méthodologie proposée pour l'inventaire du patrimoine géologique remplit le double objectif d'être conciliable avec la stratégie nationale et internationale en matière de patrimoine naturel et d'être reconnue par une majorité d'acteurs impliqués. L'État, maître d'ouvrage de l'inventaire, est représenté par ses services déconcentrés à l'échelle régionale, les directions régionales de l'Environnement (DIREN). L'inventaire sera donc conduit au niveau régional et coordonné par les DIREN. Il a été lancé officiellement en avril 2007 au Muséum national d'Histoire naturelle, en faisant le choix de lancer en priorité l'inventaire du patrimoine géologique de surface.

\section{De la théorie à la pratique}

Pour expliquer la démarche et la méthode un Vademecum a été rédigé par le Muséum national d'Histoire naturelle sous le contrôle des membres de la CPPG (De Wever et al., 2006). Le Vade-mecum, le logiciel Géotope et son guide technique sont rassemblés sur un CD-Rom gratuit disponible auprès des DIREN.

Les DIREN et les préfets de région représentant l'État sont les garants de l'exécution de l'inventaire. Les préfets de région sont chargés d'informer les collectivités territoriales, les préfets de départements, de même que les commissions départementales des sites, perspectives et paysages, et les instances départementales en charge de l'environnement quant à la phase d'élaboration de cet inventaire. L'inventaire du patrimoine naturel, placé sous la responsabilité scientifique du CSRPN, nomme un coordinateur scientifique régional. Celui-ci, entouré

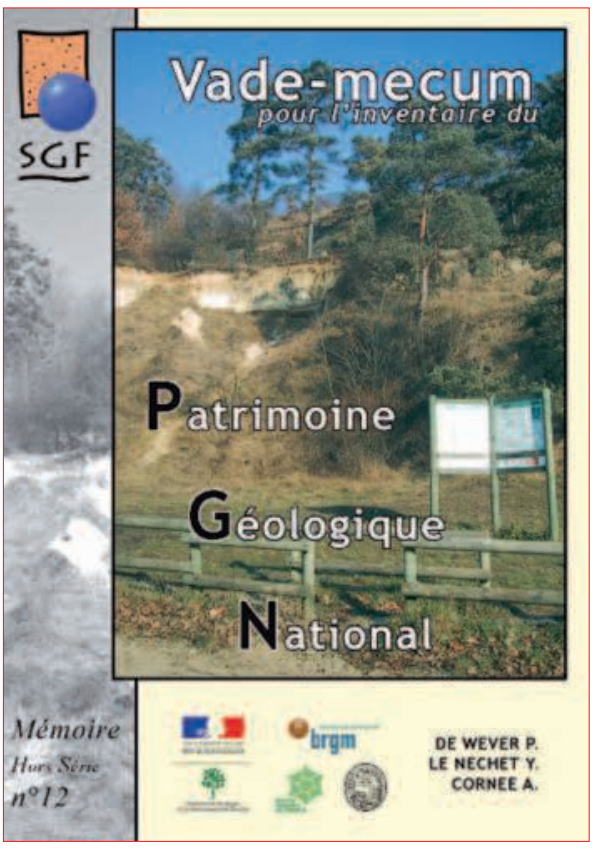

Un Vade Mecum pour l'inventaire du patrimoine géologique (C) Patrick De Wever

d'une commission régionale du patrimoine géologique, comprend un représentant du conseil régional, un représentant du CSRPN, un représentant de la DIREN, des géologues choisis selon leurs compétences (universitaires, industriels, amateurs, institutionnels...). Par ailleurs, le coordinateur scientifique régional et la commission sont aussi conviés à prendre contact avec des collecteurs potentiels, en les 


\section{L'avenir des données de l'inventaire}

Actuellement seules quelques régions ont réalisé leur inventaire, en tout ou partie. Les procédures aujourd'hui rôdées seront améliorées pour faciliter le déroulement de l'ensemble de l'opération. Après une première phase d'établissement de l'inventaire celuici devra être mis à jour puisque le statut de certains sites sera modifié, des sites disparaîtront, d'autres apparaitront au gré des modifications naturelles de l'environnement ou de l'aménagement du territoire : à cet égard un appel à participer au travail d'inventaire est adressé aux différents établissements qui conservent des collections en Sciences de la Terre, afin que les données qu'ils peuvent fournir servent à mieux apprécier l'importance de certains sites.

Par ailleurs, la base de données, telle qu'elle a été conçue, pourra s'intégrer facilement dans le réseau des global geosites du projet IUGS qui se développe en concertation avec l'UNESCO mais aussi dans un cadre plus spécifiquement européen avec le réseau ProGEO. Les données de l'inventaire seront aussi utiles pour l'aménagement du territoire. La loi (art. 411-5-1 loi 2002-276) prévoit en effet que «lors de l'élaboration d'un plan, programme ou projet, le préfet communique à la commune ou à l'établissement public de coopération intercommunale compétent toutes informations contenues dans ces inventaires utiles à cette élaboration ». Ainsi des sites ne seront plus détruits du fait de la simple ignorance comme ce fut le cas à Kersanton, en Bretagne, où une carrière, porte-nom du lithotype de la kersantite a été comblée avec des déchets ménagers, alors que cette excavation a fourni les pierres qui ont servi à sculpter tant de calvaires. Ailleurs, en Île-de-France, le niveau des Faluns de Jeurre (stampien) n'est plus visible où il fut décrit, l'endroit étant désormais occupé par un grand rond-point.

Ainsi peut-on espérer que la France, qui fut un moment pionnière en terme de patrimoine géologique puis qui a pris du retard par rapport à ses voisins retrouve une place honorable au niveau international grâce à une action menée avec la patience... d'une pierre! Comme pour élever une pyramide un long travail de base, ingrat, souterrain est à mener avant que, une fois le travail fini, on en remarque l'importance et la durabilité. Alors l'inventaire luimême sera partie intégrante du patrimoine. Le patrimoine du patrimoine!

\section{Notes}

(1) Jules Gosselet, géologue français né à Cambrai en 1832, obtint en 1864 la chaire de Géologie de l'université de Lille nouvellement créée. Fondateur en 1870 de la Société géologique du Nord, il est également à l'origine de la création en 1902 du musée de Géologie aujourd'hui rattaché au musée d'Histoire naturelle de Lille. En 1916, alors qu'il avait près de 84 ans, il continua à ranger les collections dans le musée qui, bombardé, n'avait plus de fenêtres. Il y prit froid et en mourut.

(2) Les CSRPN préexistaient mais bénéficient désormais d'un statut juridique.

\section{Bibliographie}

Billet, P. La protection du patrimoine géologique. Guide juridique. ATEN, cahiers techniques $n^{\circ} 67,2002,148 \mathrm{p}$.

De Merle, D. (coord.) Le Lutétien, collection Patrimoine géologique. Édition Muséum national d'Histoire naturelle et Biotope, 2008.

De Wever, P., Le Nechet, Y. et Cornée, A. Vade-mecum pour l'inventaire national du patrimoine géologique, Mémoires de la Société Géologique de France, hors série $\mathrm{n}^{\circ} 12,2006,162 \mathrm{p}$.

De Wever, P., Cornée, A., Reynaud, J.-Y., Vennin, E., Robin, C., Guillocheau, F. et Rouby D. Des stratotypes au Bassin de Paris : deux siècles de stratigraphie, in Alcide d'Orbigny, du Nouveau Monde... au passé $d u$ monde. Éditions Nathan et Muséum national d'Histoire naturelle, 2002, pp. 89-99.

Martini, G. (coord.) Actes du premier symposium international du patrimoine géologique, Digne-les-Bains, 11-16 juin 1991, Mémoires de la Société Géologique de France, n¹65, 1994, 276 p. 